\title{
Atomic resolution investigation of electric field effects on equilibrium grain boundary configurations in ceramics
}

\author{
Lauren A Hughes ${ }^{1,2}$, Klaus van Benthem ${ }^{1 *}$ \\ 1. Department of Materials Science and Engineering, University of California, Davis, Davis, CA 95616, \\ USA \\ 2. National Center for Electron Microscopy, Lawrence Berkeley National Laboratory, Berkeley, CA \\ 94720 USA
}

* Corresponding author: benthem@ucdavis.edu

The joining of ceramics is an important technology for the formation of new components with desired properties, including wear resistant surfaces, ultra-hardness in cutting tools, corrosion resistance, high temperature protection, and electrical or ionic conductivity. A fundamental concept of joining is sintering of nanoparticles. Electric field assisted sintering, which includes spark plasma sintering and flash sintering, has shown that the application of electric fields during materials processing can cause accelerated densification and grain growth. The atomic-scale mechanisms for grain boundary formation, however, remain unknown.

Bicrystals of $42^{\circ}(100)$ twist grain boundaries were formed by diffusion bonding at high temperature in air. During diffusion bonding electrostatic fields with varying field strengths were applied across the forming grain boundary plane (see sketch in Figure 1). Cross-sectional TEM specimen preparation was subsequently carried out by focused ion beam sectioning using a FEI Scios dual-beam FIB. Atomic and electronic interface structures were subsequently determined from high-angle annular dark-field (HAADF) images and energy-loss near-edge fine structures (ELNES) acquired with a spherical aberration-corrected Jeol JEM 2100F/Cs scanning transmission electron microscope (STEM).

Grain boundary core structures formed in the presence of an applied electrostatic field strength of nominally $20 \mathrm{~V} / \mathrm{cm}$ show an abrupt and atomically structured interface with an interface expansion of $0.43 \pm 0.03 \mathrm{~nm}$ (Figure $2 \mathrm{a}$ ). In the absence of an applied electric field strength, however, the grain boundary core structure revealed a width of $0.89 \pm 0.13 \mathrm{~nm}$ (Figure 2b) [2]. Further increase of the nominal field strengths to values as high as $170 \mathrm{~V} / \mathrm{cm}$ revealed no obvious changes in the cationic interface configuration [3].

ELNES analysis of grain boundary cores formed under $20 \mathrm{~V} / \mathrm{cm}$ reveal local electronic structures that suggest the presence of distorted $\mathrm{TiO}_{6}$ octahedra, while those formed in the absence of any electric field show bonding configurations similar to reduced $\mathrm{SrTiO}_{3}$. Further increase of the applied electric field strength, however, indicates potential disordering of the oxygen sub-lattice, as the local unoccupied densities of states are similar to those of amorphous bulk $\mathrm{TiO}_{2}$ [3]. The observed interface configurations are attributed to electric field-induced defect concentration gradients in the free (100) $\mathrm{SrTiO}_{3}$ surfaces prior to diffusion bonding. Additional experimental studies are underway to explore the thermodynamic stability of the observed grain boundary core structures, and how electric field effects may change for grain boundary planes different from (100).

References:

[1] R.H.R. Castro, K. van Benthem. Sintering. Springer Science \& Business Media; 2012.

[2] L.A. Hughes, M. Marple, K. van Benthem. Appl Phys Lett. 113 (2018) 041604. 
[3] L.A. Hughes, K. van Benthem. J. Amer. Ceram. Soc. (2019) in press.

[4] LAH acknowledges financial support through US National Science Foundation Graduate Research Fellowship Grant \#1650042. The majority of the project was financially supported by the Army Research Office under contract W911NF-16-1-0394 (program manager Dr. Michael Bakas). The latest experimental results were funded by the National Science Foundation under award DMR-1836571. Work at the Molecular Foundry was supported by the Office of Science, Office of Basic Energy Sciences, of the U.S. Department of Energy under Contract No. DE-AC02-05CH11231.

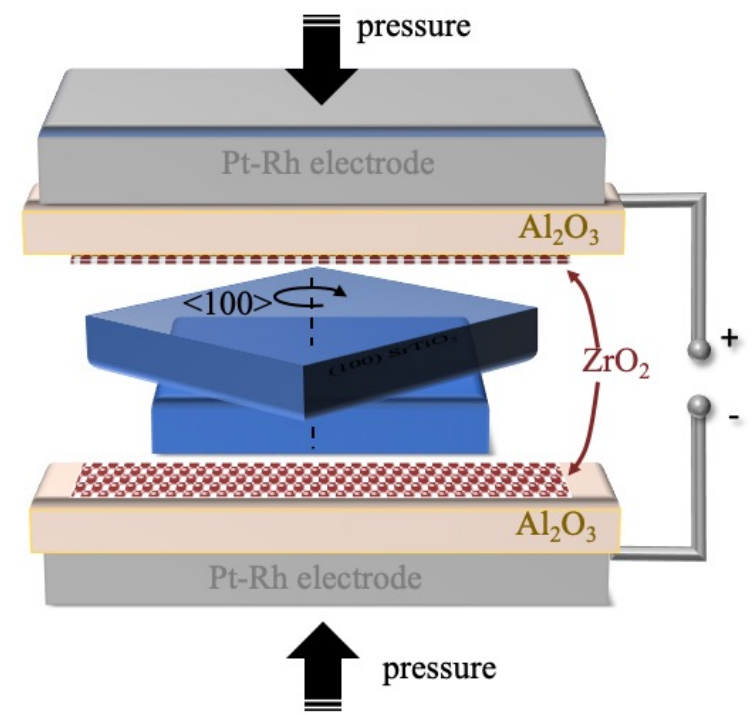

Figure 1. Sketch of the experimental setup for diffusion bonding under simultaneously applied electrostatic fields.
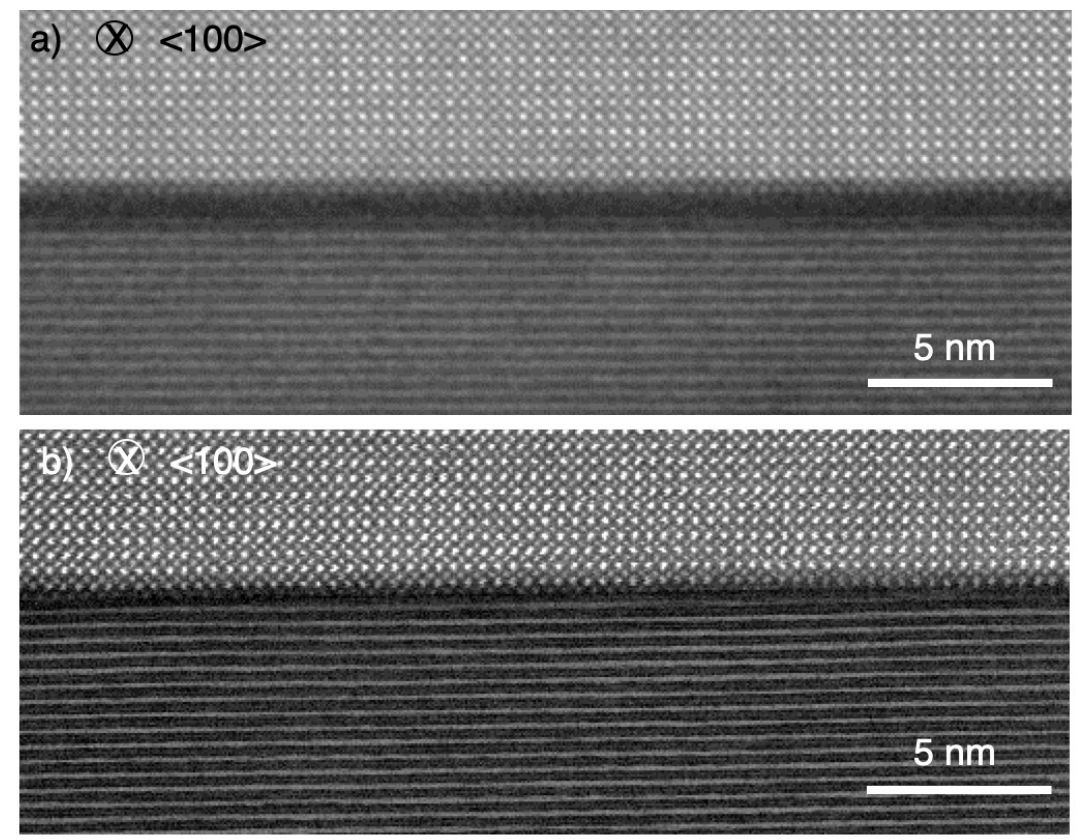

Figure 2. HAADF-STEM images of (100) twist grain boundaries formed by diffusion bonding in (a) the presence, and (b) the absence of an applied electric field strength of nominally $20 \mathrm{~V} / \mathrm{cm}$. 